\title{
No limbits! \\ The feasibility of providing low-cost 3D printed below elbow and below knee limb replacements in a resource limited setting
}

Te Water Naude M. (matt.tewater@gmail.com)

Roussot M. A. (markroussot@gmail.com)

Sweatman K. (SWTKEL001@myuct.ac.za)

Gabier R. (GBRRUQ001@myuct.ac.za)

Supervisor: Dr Mark Roussot

Division of Orthopaedic Surgery, University of Cape Town

\begin{abstract}
Background: South Africa faces the challenge of producing large numbers of prostheses each year. Three-dimensional (3D) printing was investigated as a potential method of efficiently providing prostheses.
\end{abstract}

Objective: To evaluate the need for below-knee (BK) and below-elbow (BE) limb replacements at Groote Schuur Hospital; and to determine the feasibility of providing low-cost 3D printed limb replacements at Groote Schuur Hospital (GSH).

Methods: We performed a literature review and conducted interviews with staff at the Amputation clinic. Information regarding the need for $B E$ and $B K$ prostheses, qualifying criteria, durability and cost of traditional prostheses, and manufacture times was collected. Communication with e-NABLE and a senior biomechanical engineer provided further information. We investigated the feasibility of producing a 3D printed prosthesis by printing and assembling a prosthetic hand.

Results: 3D printing reduces the cost of a BE prosthesis by approximately $26.6 \%$ and shortens the manufacturing time; however, 3D printing technology is currently unable to provide adequate weight bearing $B K$ prostheses and is not as cost efficient as traditional manufacturing techniques.

Conclusion: It is feasible to provide $3 D$ printed BE prostheses in a resource-limited setting; however, BK prostheses face design challenges and are more costly than those traditionally made.

Keywords: Below-knee amputation, below-elbow amputation, prosthesis, 3D printing. 


\section{Introduction}

South Africa (SA) is burdened with producing high numbers of prostheses each year. The Orthotics and Prosthetics Centre in Pinelands (which services the Western Cape) produces approximately 3000 prostheses annually (Nel, personal communication 2015, 27 July). Globally, amputations occur as complications of diabetes, vasculopathy and trauma (FrederiksI \& Visagiell, 2013). Currently, there is little information available on amputation rates and causes as well as prosthesis production in SA. Due to the high cost of prostheses offered locally ( $\mathrm{Nel}$, personal communication 2015, 27 July), there is a need to investigate lower-cost possibilities in prosthesis production. Children have the added disadvantage of outgrowing their limbreplacements which further exponentiates the cost ( $\mathrm{Nel}$, personal communication 2015, 27 July).

Traditional prosthesis fabrication requires the formation of a socket that is manually customised by a well-trained technician for each amputee, specific to the geometry of his or her residual limb (Herbert et al., 2005). However, the process is labour intensive (Francis et al., 2002), involves casting and moulding of the residual limb (Herbert et al., 2005), and is time consuming for the amputee. This is a process that is thought to contribute to the psychological impact of obtaining a prosthesis (Colombo et al., 2010). Further, the costs to create and maintain a prosthesis are high (Zuniga et al., 2015), placing a large burden on the public sector resources. This approach leaves no permanent record of the residual limb as casts and moulds are destroyed during final socket fabrication, resulting in the entire process of casting through to fabrication being repeated when an old prosthesis has to be replaced (Herbert et al., 2005).

Advancements in 3D scanning and 3D printing technologies are opening doors for faster and fully automated prosthesis production that accurately match the socket to patient anatomy (Francis et al., 2002; Rahmati, Farahmand \& Abbaszadeh, 2011) while at the same time increasing the scope for digital storage and modification of prosthetic socket designs (Herbert et al., 2005; Francis et al., 2002; Colombo et al., 2010; Smith \& Burgess, 2001). 
It has been demonstrated that a 3D-printed upper-limb prostheses can be produced for US\$50, which equates to a cost reduction of 80 times when compared to the traditional method, which costs US\$4000 (Zuniga et al., 2015). This large reduction in the cost is a major benefit in a resource-limited setting and helps to make the saved resources available for use in other areas. The process of final adjustments to the gait of the patient must still be conducted manually by a trained prosthetist after fitment of the prosthesis, as with conventional prostheses (Rogers et al., 2007).

There is currently no literature reporting on amputation rates in South African hospitals, and the magnitude of the burden of providing limb replacements in this setting remains unknown. To our knowledge no studies have evaluated the need for BK and BE limb replacements or determined the feasibility of providing 3D-printed limb replacements in South Africa

\section{Objectives}

To evaluate the need for $\mathrm{BK}$ and $\mathrm{BE}$ limb replacements at GSH; and determine the feasibility of providing low Cost 3D printed BK and BE limb replacements at GSH.

\section{Methods}

This is a feasibility study conducted at GSH in 2 phases.

\section{Phase 1}

\section{Research Procedures and Data Collection Methods}

Information was collected in the form of interviews with the prosthetist in charge of the Amputation clinic at GSH and the Orthotics and Prosthetics centre. A Physiotherapist involved at the amputation clinic at GSH was also interviewed. The interview with the prosthetist was recorded and transcribed. Permission was not granted to record the physiotherapist's interview however notes were taken.

The books for the amputation clinic at GSH were reviewed for the period January to July 2015 in order to quantify the number of BK and BE amputees that attend the clinic as well as the cause for their amputation. 
The demand for limb replacements was evaluated along with their qualifying criteria, function and durability, waiting times, challenges in rehabilitation, different designs and design cost.

The cost-list given to the unsubsidized patients was used as it is believed that this represents the cost of manufacturing the prosthesis (Gretsch et al., 2015).

\section{Phase 2}

\section{Research Procedures and Data Collection Methods}

The e-NABLE website containing freely available information from types of 3D printers to different prosthetic devices, their printing instructions and assembly guides were downloaded and thoroughly examined.

Consultations with experts from different 3D scanning and printing companies and staff in the UCT Engineering department were held. Quotations were obtained from various 3D printing companies via email and bot correspondence.

Contact was established with 3D Universe to decide on the most feasible option to acquire hardware needed in assembly of prostheses. Hereafter local hardware stores were contacted.

A demonstration Cyborg Beast was printed and assembled using the profits of selling 3D printed necklaces.

The above methods were used to obtain information on:

1. Process of creating 3D prosthesis

2. Functionality and durability

3. Requirements and cost

\section{Results}

\section{Phase 1}

\section{The need for BK and BE prostheses}

At The Orthotics and Prosthetic Centre they often make up to three BK prostheses per day, whereas the need for BE prostheses is less. This year there have 
been very few but for the prior two and a half years there have been up to forty per year (Nel, personal communication 2015, 27 July).

Twenty six BK amputees, and no BE amputees, attended the Amputation Clinic at GSH for the period January to July 2015.

\section{The causes of BK amputations at the Amputation Clinic at GSH}

Of the 26 BK amputations reviewed $69 \%$ were of vascular causes and $23 \%$ were of traumatic causes (Figure 1). Information collected from the books for the Amputation Clinic at GSH.

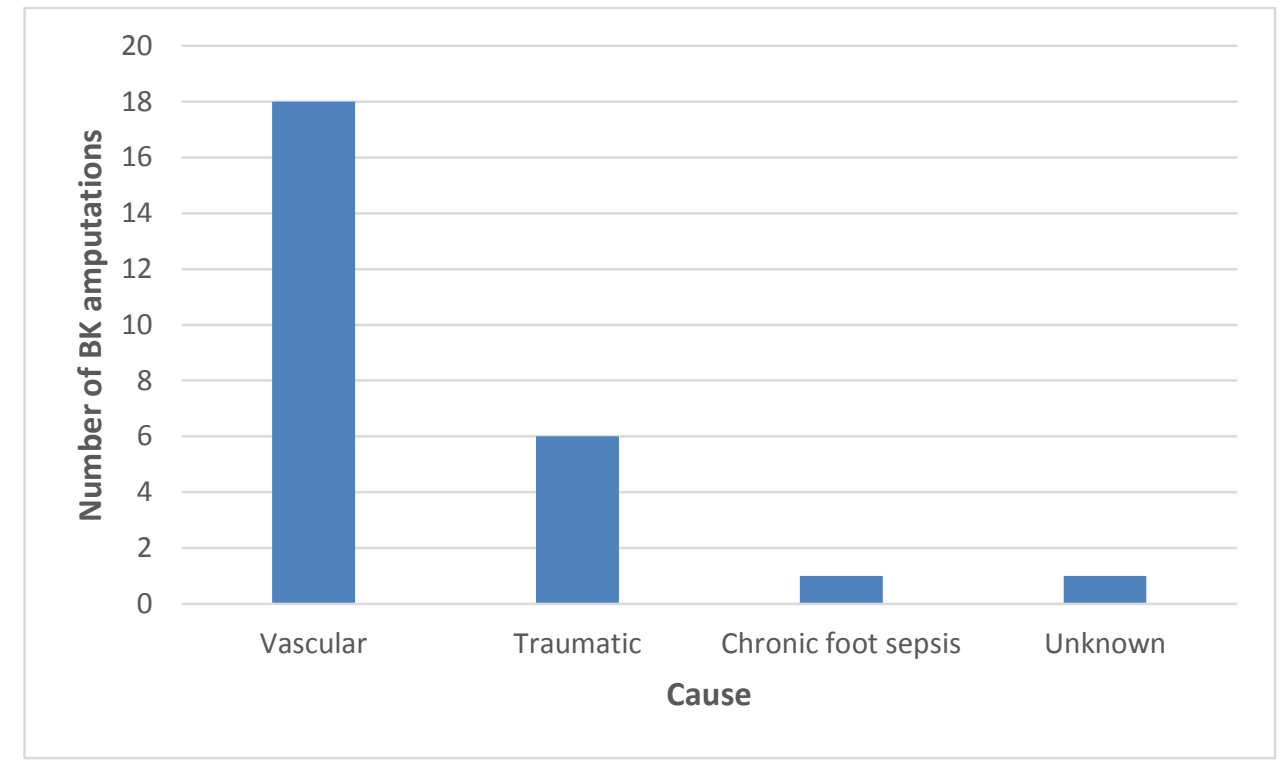

Figure 1: The causes of BK amputations as seen at the Amputation Clinic at GSH $^{*}$

${ }^{*}$ Of these amputations a bilateral (vascular) case was recorded

** Information collected from the books for the Amputation Clinic at GSH

\section{The criteria that qualify a patient for prosthesis}

There are many criteria for lower limb amputees. Some of the important criteria include: the residual limb must be cone-shaped, there must be no swelling, full range of motion should be achieved and the scar should be mobile (Anon., 2015). The patient should also be using crutches with ease, not using a wheelchair and should be in generally good health (Nel, personal communication 2015, 27 July). 
The proportion of patients that meet the criteria and receive prosthesis and those who do not

According to the prosthetist, all patients that meet the criteria receive a prosthesis (Nel, personal communication 2015, 27 July).

\section{The durability of BK and BE prostheses}

BK prostheses should last four to five years. The exoskeleton legs do last that long however the modular designs tend to last for two and a half to three years. The amputee's first lower limb prosthesis needs to be replaced after six months after the soft tissue swelling has resolved (Nel, personal communication 2015, 27 July). BE prostheses, due to the fact that they are not weight bearing, can last for up to ten years (Nel, personal communication 2015, 27 July).

Many other adverse conditions can shorten the life-span of a prosthesis such as a harsh environment (Anon., 2015).

\section{The time-frame in which children outgrow, and therefore need new prostheses}

This is variable and depends on the growth rate of the specific child. Often prostheses are made slightly bigger so that they fit with one stump sock which ensures a longer time-frame of use. It should last for roughly one year (Nel, personal communication 2015, 27 July).

\section{The time frame from being approved for prosthesis to receiving it}

The patients receive their first appointment within two weeks for casting and measurements. For BK prosthesis, between one to two weeks later the prosthesis will be ready. The patient will be fitted and can walk out the door with the prosthetic limb on the same day (Nel, personal communication 2015, 27 July).

With the BE prosthesis it takes longer. The parts are imported from Germany which can take up to three to six months (Nel, personal communication 2015, 27 July).

\section{The rehabilitation process for $\mathrm{BK}$ and $\mathrm{BE}$ amputees that receive prosthesis}

For BK amputees, the main focus is walking. It is important to walk on different surfaces and in different directions, on slopes and up stairs. The aim is to eventually 
walk without the crutches. At GSH, PPAM aids (pneumatic post-amputation mobility aid) are used to facilitate this process (Anon., 2015).

For the upper limb, the focus is more with the occupational therapists however the physiotherapists focus on getting the shoulder and elbow joints back to having a full range of motion (Anon., 2015).

\section{The most common complaints by patients}

The most frequently reported complaint is that the prosthesis is too heavy while some complain that it does not fit comfortably (Nel, personal communication 2015, 27 July; Anon., 2015).

How regularly people fitted for prosthesis do not collect their completed prosthetic limb

It happens frequently with arms but seldom with lower limbs (Nel, personal communication 2015, 27 July).

\section{The current different designs for BK and BE prostheses used in the public} sector

There are two types of BK prosthetic designs - the PTS (Patella Tendon Supracondylar) and the PTB (Patellar Tendon Bearing). They are named according to how they attach to the residual limb - via a strap or pressure points. They can both either be a hard leg (exoskeleton) or modular (endoskeleton) (Nel, personal communication 2015, 27 July), see Figure 2. Only one design for BE prosthesis is used - the static, aesthetic arm (Nel, personal communication 2015, 27 July).

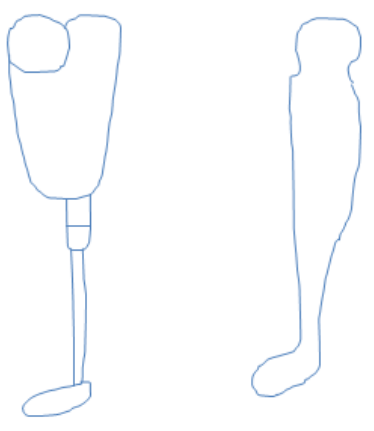

Figure 2: The modular (Left) vs. exoskeleton (Right) prostheses 


\section{How the different designs effect the rehabilitation}

According to the prosthetist, the prosthesis that will suit the patient best is used and therefore it won't affect the rehabilitation process (Nel, personal communication 2015, 27 July). Having a lighter prosthesis could improve the rehabilitation process according to the physiotherapist. The weight of the prosthesis is especially important in cardiac patients (Anon., 2015).

\section{D printing in the future}

Both the prosthetist and physiotherapist were happy to adapt and learn about 3D printing (Nel, personal communication 2015, 27 July; Anon. 2015). There was some doubt with regards to the cost effectiveness form one perspective (Herbert et al., 2005), but on the other hand there was excitement and enthusiasm (Francis et al., 2002).

\section{The costing of the prostheses}

According to the prosthetist, for a BK prosthesis, the kit ordered is roughly R1400. If labour, casting, materials such as fibreglass and plaster work are added, the total would be around R3400 (Nel, personal communication 2015, 27 July). The unsubsidised cost is R4033 for the PTB/PTS. The PTB/PTS legs come with two stump socks and two sheaths which cost R111 each. With regards to the arms, the estimated manufacturing cost is R8000 (R4000 for the glove and R4000 for the hand) (Nel, personal communication 2015, 27 July). The unsubsidised cost, is R8064.

In summary, the below knee prosthesis is either a PTS or PTB, will costs R4033 and is expected to last between 4 and 5 years. The BE prosthesis is a static arm with no functional capacity, costs R8064 and is expected to last 10 years.

\section{Phase 2}

\section{The process of making 3D printed prostheses}

The basic steps to print a prosthesis includes: preparation, printing and assembly. A 3D printed prosthesis consists of printed parts and hardware. The printing process used is called Fused Deposition Modelling or Fused Filament Fabrication which show to be the most feasible option (e-NABLE, 2015). 
There are two options to obtain plans for a 3D printed limb: Designs are freely available online licensed under the Creative Commons - Attribution - Non-Commercial license on the e-NABLE website (e-NABLE, 2015). Links are present to direct the user to the source such as thingserse.com among other websites which offer the option to download the 3D printing plans (.stl files), instruction manual and assembly guide of a specified prosthetic device at no cost.

Option two is to approach an engineer who will cost $\sim$ R1000/hour to design a prosthesis. The planning and design roughly take 100 working hours, according to an engineer at our university, who is involved in the forefront of 3D printing prostheses and implants in South Africa. This cost is once off cost as the design will be repeatedly used.

\section{Hardware}

In addition to the 3D printed parts, hardware is required. These parts are not yet printable. It includes screws, braided fishing line, tensioners etc. These can be sourced from international online store, 3Duniverse (2015). However it is more convenient and cost effective to source from a local hardware store.

\section{Assembly process}

The assembly process can be completed within two hours and does not require a specifically trained person. The above mentioned time taken can be improved by holding workshops to build fine motor skills. Alternatively the assistant can be paid a flat rate per prosthesis instead of working hours logged.

\section{Below elbow}

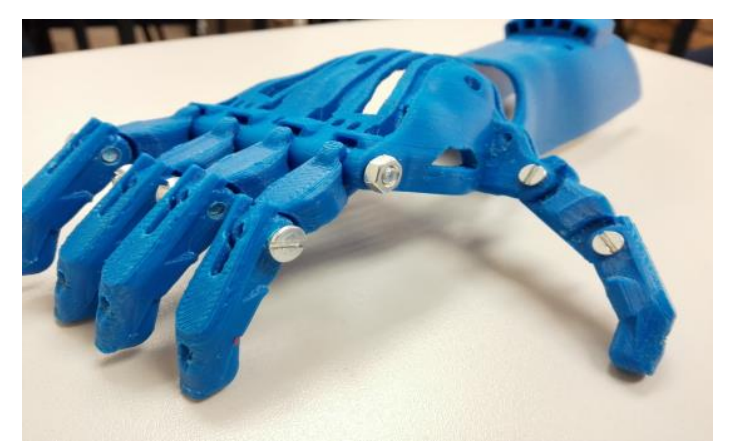

\section{Cyborg Beast}

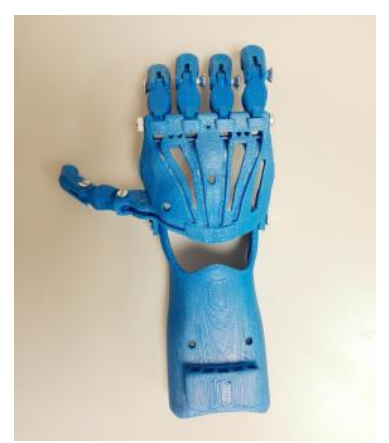

Figure 3: The Cyborg Beast (e-NABLE, 2015) 
The Cyborg Beast requires the recipient to have a functional wrist and strength to provide sufficient functional capacity (e-NABLE, 2015). It is not a replacement for a fully functional arm, however does provide the ability to grasp objects which are relatively light and pliable (e-NABLE, 2015). Elastic cords placed through the dorsal channels of the proximal and distal fingers cause passive extension of fingers. Flexing the fingers is achieved by non-elastic cords on the palmar surface channels of each finger. This process is set in motion by $20-30^{\circ}$ of wrist flexion. The limitation of this design is it not being suitable for children under the age of three due to the increased risk of falling (e-NABLE, 2015).

The customisation of parts can be achieved by using the scaling process through the 'Handomatic' programme (e-NABLE, 2015). Measurements which can be done physically or via the use of photos of residual limb are taken and entered into the programme. An algorithm runs and produces the correct dimensions of parts to be unique to the residual limb, ultimately resulting in a better fit.

Using the quotations obtained a standard child sized Cyborg Beast would cost R1274.25 (See Table 3).

The relationship between size of prosthesis and cost of printing can be explained using the scaling chart e-NABLE (2015) provides. It is used to determine the scaling factor for people different ages. Protolink3d (Havinga, personal communication 2015, 30 July) assisted in using this scaling chart to determine the costing for prints of different sizes catering for people of various ages. The scale chart goes to age 16 which requires parts to be scaled by $40 \%$. A $50 \%$ scaling factor caters for the size of an adult.

The effect of scaling for adults results in a volumetric increase from $63 \mathrm{~cm}^{3}$ to $203 \mathrm{~cm}^{3}$ and print time is increased by three fold, i.e. three child sized prostheses can be printed for the cost of an adult sized prosthesis. The price increases due to increase in printing time as the parts for an adult prosthesis are larger. The resultant prince increase is 2.4 fold, thus the cost of a standard adult sized Cyborg Beast is R2306 (See Table 3).

As part of this feasibility study, a child sized Cyborg Beast was printed. Costs were fully covered by the selling of 3D printed Lotus Flower necklaces both privately 
and at the UCT second year medical students' Special Study Module Exhibition. Necklaces were printed by Laurens' Hub (Weyn, personal communication 2015, 3 August). The cost per necklace was R17 and sold for R50, producing a R33 profit each. 39 necklaces were sold to individuals who were willing to 'Give a Hand'. The total profit of $\mathrm{R} 1289$, was used cover the prosthesis cost (R1274.25) indicative a selfsustaining process. For every 39 necklaces sold, a child sized Cyborg Beast can be produced 'for free'. To fund an adult sized Cyborg Beast, 63 necklaces need to be sold.

Table 1: Cost of printer and print/hour for cyborg beast

Decent 3D printer: R25 000 (needs to be paid within 5 years)

Printing/Hour:

Printer Cost $\quad$ R25 000 (Protolink3d, 2015; 3D Printing Systems, 2015).

Runs for 8 hours a day

5 days per week

10 months

5 years

25000/2000 hours $=$ R12.50/hour

Maintenance R100 per print job

Labour (Trained 3D printer technician)

R50/hour

TOTAL PRINTING COST/HOUR

R162.50 (R2.70/min)

Table 2: Cost for child sized cyborg beast using R25 000 printer

Mass of ABS needed: 44.6g@ 90c/g R 40.14

Print time: $376 \mathrm{~min}$

R 1018.00

Hardware R 341.00

Assembly time $<2$ hours

$\mathrm{R} 100.00$

Total cost

R 1499.14 
Table 3: Cost for cyborg hand using Protolink3D

\begin{tabular}{|c|c|c|}
\hline & Child & Adult \\
\hline Printing and materials & R 758.25 & R 1790.00 \\
\hline Set up fee & R 75.00 & R 75.00 \\
\hline *Hardware & R 70.00 & R 100.00 \\
\hline Assembly time $<2$ hours & R 100.00 & R 100.00 \\
\hline Total cost & R 1003.25 & R 2065.00 \\
\hline
\end{tabular}

* Cost for kit by 3D Universe (2015) = \$35 (kit) + \$69 (shipping) or individually sourced from local hardware stores in Cape Town costing a sum total of R100.

\section{Rochester Institute of Technology (RIT) ARM}

The RIT Arm requires the recipient to have a functioning elbow and allows for basic functions such as picking up a bottle or catching a ball, it cannot sustain more than a few kilograms (3Duniverse, 2015).

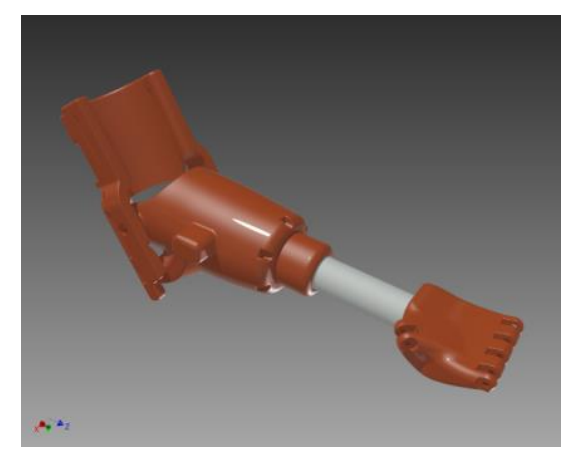

Figure 4: The RIT arm by e-NABLE (http://enablingthefuture.org/upper-limbprosthetics/rit-arm/)

This model is intended for transradial amputees. The above-elbow gauntlet is hinged (A) to a forearm cup, the forearm cup (B) has a socket at the proximal end and connects distally, through a hand rotation mechanism (C), to a PVC pipe (D). The PVC pipe secures the hand (E) (e-NABLE, 2015). The bending of the elbow causes the fingers to flex by tightening strings attached to the fingers from tensioners in the upper arm. 
Using the quotations obtained the standard child sized RIT Arm would cost R2824.87. Using the scaling chart the calculated cost for an adult sized RIT Arm is R6162.29 (Refer to table 4).

Table 4: Cost for RIT arm using Protolink3D

Child Adult

\begin{tabular}{r|l|l|}
\hline Print time: 54.3 hours & $R 2383.87$ & R 5721.29 \\
Hardware & R 70.00 & R 100.00 \\
\cline { 2 - 3 } Assembly time < 2 hours & R 100.00 & R 100.00 \\
Total cost & R 2553.87 & R 5921.29 \\
\cline { 2 - 3 } & &
\end{tabular}

\section{Below knee}

Currently, there are no open source plans available to 3D print BK prostheses. This is due to their weight bearing nature, a factor which need not be considered in the BE prosthesis. The Roboleg (Robohand, 2015) which features a functional ankle and knee joint is an almost entirely 3D printed leg by pioneers Richard van As and Ivan Owen has shown promising results. Final adjustments are still in progress before being released as open sourced plans. It is recommended that further research on feasibility is conducted once this is released.

A proposed solution is to print parts of a BK prosthesis. One such example is to use 3D scanning technology in conjunction with 3D printing to produce a customised socket catering for each contour of the residual limb. The specific socket seen below has been designed by Redline Laser (Erasmus, personal communication 2015,24 July) specifically for use in this study. It is a model including a 316 stainless steel socket secured by $5 \times 5 \times 25 \mathrm{~mm}$ cap screws. However, it is not a validated design and further investigation is needed. 

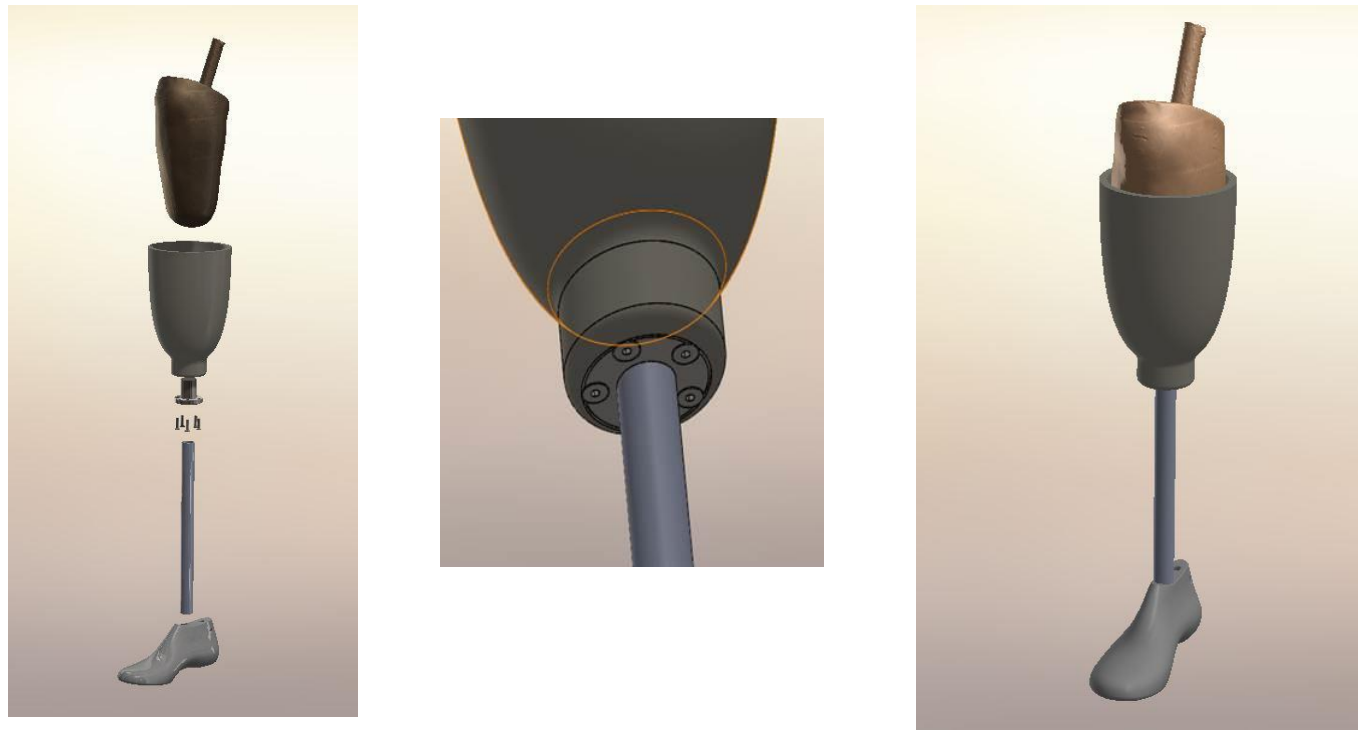

Figure 5: Scans of BK socket (Erasmus, 2015)

The cost of a BK socket from Protolink3d (Havinga, personal communication 2015, 30 July) could not be obtained as they cannot accommodate the printing of stainless steel. Further investigation is required to determine the cost of obtaining a stainless steel printer, and the cost of printing per hour.

Table 5: Comparison of costs of current vs. 3D printed prostheses for adults

Current*

(R)
3D printed*

(R)
Price difference

(\%)

\begin{tabular}{|r|l|l|l|} 
Below elbow & 8064 & 5921.29 & -26.6 \\
\cline { 2 - 4 } Below knee & $4033-5508$ & inconclusive & NA \\
\cline { 2 - 4 } & &
\end{tabular}

* Information received from unsubsidized cost list

${ }^{* *}$ Refer to table 5

In summary, the BE (RIT arm) cost is R5921.29. The below wrist (Cyborg Beast) cost is R2065 this can be funded by selling 63 necklaces. The BK cost is inconclusive and their durability is yet to be determined. 


\section{Discussion}

There is a large demand for prostheses in South Africa, mainly due to the high prevalence of diabetes as well as trauma related injuries in our population ( $\mathrm{Nel}$, personal communication 2015, 27 July; Anon., 2015) The Prosthetic and Orthotic Centre in Pinelands (which serves the Western Cape) is required to produce close to 3000 prostheses per year (Nel, personal communication 2015, 27 July). During this study we were able to demonstrate that it is feasible and cost effective to provide $3 \mathrm{D}$ printed BE prostheses in a resource-limited setting; however, BK prostheses still face design challenges and are relatively more costly than those that are made manually.

The BE prostheses offered are non-functional, cost approximately R8064 and last up to 10 years (Gretsch et al., 2015). In contrast, a 3D printed BE functional prosthesis (RIT arm) costs R5921.29 (R100 provision for hardware included), which is approximately $26.6 \%$ less than the current cost, although its durability remains uncertain. The imperial sized screws suggested by e-NABLE cost $\$ 104$ (shipping included), but by sourcing the nearest equivalent locally and using metric sizes to obtain the best functionality and reduce assembly time, the cost was decreased by approximately $92.4 \%$. 3D printing technology can produce five prostheses for every four produced currently.

The cyborg beast, which needs a functional wrist, costs a total of R2065. Currently no options are available for below wrist amputees, however, the throughwrist unsubsidized cost is R16287.

In contrast to the current non-functional BE prostheses, the prostheses at eNABLE (2015) allow for basic functions such as lifting objects. In addition to the cyborg beast and the RIT arm, a higher functioning arm created at UCT, has further increased functionality and is currently produced for R11500 (Vicatos, personal communication 2015, 30 July) only R3436 more than the current prostheses.

Currently if a patient requires a functional "hand", they are supplied with a dynamic hook, a far less aesthetically pleasing and considerably more expensive option at R8000 - R15000 (Gretsch et al., 2015) per hook, becoming up to between R16064 and R23064 when attached to the BE prosthesis. 
Although the prosthetist did not think that the need for BE prosthesis would increase by adding a standard dynamic option (Nel, personal communication 2015, 27 July) the physiotherapist seemed to think it would greatly increase the need (Anon., 2015) and in any case, it seems that the additional functionality could have a positive impact on amputee's lives.

A possible disadvantage of the $3 \mathrm{D}$ printed $\mathrm{BE}$ prostheses is, as previously mentioned, the uncertainty on the durability (e-NABLE, 2015). Aesthetically, they also do not resemble human hands and some may say they are "robotic-looking". Due to the growth rate of children, their prostheses need to be replaced once every year which exponentiates the costs involved ( Nel, personal communication 2015, 27 July). The cyborg beast hand and RIT arm, being a quarter of the cost, cater for this need.

When patients meet the criteria for a $\mathrm{BE}$ prosthesis, they can be measured immediately. However due to the fact that the parts must be imported from Germany it takes up to 6 months for their delivery. With the evolution of 3D printing, all the parts could be produced and sourced locally eliminating this waiting period. They could be printed within one day and assembled in less than two hours (e-NABLE, 2015).

It is evident that 3D printing can be self-sustainable. As part of this feasibility study, a child sized Cyborg Beast was printed (Havinga, personal communication 2015, 30 July) and the costs were covered by selling 39 3D printed necklaces. This number can be easily reduced as printing in bulk decreases the cost of both the necklace and the prosthesis.

The current BK prostheses offered are PTS or PTB. They are expected to last between two and a half and five years with the unsubsidized cost being R4033 R5508 ( Nel, personal communication 2015, 27 July). There are no open-source plans currently available for BK prosthesis. For this reason we approached RedlineLaser who used 3D scanning to create a ".stl" file to print a part of a BK prosthesis - the socket. The most suitable material is stainless steel however the quote obtained uses PLA due to limitations on Protolink3D's printer and costs R1384.90 (Havinga, personal communication 2015, 30 July). ABS (used in the BE prosthesis) is stronger than PLA but is not suitable for BK prosthesis as it warps on large scale. Getting a quote for stainless steel prostheses remains a challenge as the printers are difficult to source 
and are expensive. There is however a printer costing R6million which can print in any material (Vicatos, personal communication 2015, 30 July).

Printing a BK prosthesis would require scanning of the residual limb, while BE would just require a few simple measurements (Francis et al., 2002; Rahmati, Farahmand \& Abbaszadeh, 2011). This is due to its weight bearing capacity. Transtibilal prostheses would also need to fit correctly to ensure that adequate blood supply is maintained and that forces are correctly distributed (Colombo et al., 2010). Scanning adds an extra cost to the BK prosthesis, however the scanning of the residual limb presents the advantage that the files can be stored for future use if another prosthesis is required.

For BK prostheses, once a patient has met the criteria, an appointment is scheduled within two weeks for casting and measurements. This would remain the same if the prosthesis were 3D printed. Hereafter, eight hours are required for manual manufacture. The patient will then receive it within a further two weeks.

The 3D printing of a socket in PLA (which prints faster than stainless steel) would take 26 hours Erasmus, personal communication 2015, 24 July). An additional two hour period is needed to assemble all the parts. A printer can only print one socket at a time despite the high demand for BK prostheses; currently up to three BK amputees are seen per day (Nel, personal communication 2015, 27 July). Three to four printers constantly running would be required just to keep up with the current demand. Three traditionally made prostheses can be manufactured in the time that one socket is printed; and the BK prosthesis would still need to be assembled. This is a further limitation of 3D printing.

With regards to the rehabilitation process, and especially considering cardiac patients who may struggle to cope with the new weight of the prosthesis, it is thought that a lighter material could help this process (Anon., 2015) however it must be noted that the current prostheses are in fact lighter than an actual leg would be - weighing a maximum of $3 \mathrm{~kg}$ ( $\mathrm{Nel}$, personal communication 2015, 27 July). It could therefore be recommended that more research is done into whether a lighter, more user friendly material would improve the rehabilitation process. 
It appears evident that the quantitative need for BK prostheses is greater than that of BE (Nel, personal communication 2015, 27 July). Although patients that meet the criteria receive a prosthesis, there is a filtering process and many do not reach this stage. There is scope for many more prostheses to be granted and 3D printing could facilitate this process. Research should be done on how to improve the number of patients that reach the prosthetist.

3D printing can reduce the time spent for skilled professionals and in so doing can make prosthetic services more accessible (Colombo et al., 2010). It has been shown that 3D printed prostheses can be produced for 80 times less than the traditional prosthesis (Zuniga et al., 2015).

In contrast, 3D printing has limitations that require further research, especially to improve the CAD software as well as the scanning technology to prevent scan alterations if the amputee changes position (Colombo et al., 2010; Rogers et al., 2007).

\section{Conclusion and recommendations}

This study has demonstrated that it is feasible to produce 3D printed BE prostheses that are more functional and cost effective than prostheses that are currently available in our setting, and can be fabricated in a shorter period of time. While formal research on durability is required, 3D printed BE prostheses are expected to have similar longevity to those made by traditional methods.

Conversely, 3D printed BK prostheses still face numerous challenges that encumber their feasibility in our setting. These challenges include the lack of open source designs, difficulties with customization of fit to the lower limb stump and weight bearing requirements. Therefore, traditional methods of fabricating BK prostheses are still recommended.

While this study was unable to quantify the amputation rate and demand for prosthetic limbs at Groote Schuur Hospital during the available time, it was found that the Western Cape Rehabilitation Centre fabricates approximately 3000 prostheses annually. This equates to an annual expenditure upward of R12, 000,000. Employing the use of $3 \mathrm{D}$ printing technology, and thereby improving cost efficiency, as 
demonstrated in our evaluation of BE prostheses, could potentially save our Western Cape Health Care system up to $26 \%$ on the BE prostheses annual budget.

Future research is required to:

1. Determine the amputation rates (BE versus $B K$ ) and quantify the burden of prosthesis provision at Groote Schuur Hospital for the period 1995-2015.

2. Critically analyse the materials and methods best suited for 3D printing prosthetic limbs,

3. Evaluate the durability of 3D printed limb replacements; and

4. Develop a reproducible, open source design for a 3D printed BK prosthesis that is customizable in terms of fit.

5. Increase the percentage of patients seen by the prosthetist

To our knowledge, this is the first study to evaluate the feasibility of 3D printed limb replacements in a resource-limited setting.

\section{Acknowledgments}

Dr George Vicatos for giving us insight into the world of 3D printing prosthetics.

Mr Marc Erasmus for providing us with information on scanning and 3D printing.

Ms Olwen Nel for sharing her knowledge of current prosthetic limbs.

Mr Martin Havinga for printing the Cyborg Beast hand and providing cost price quotations. Furthermore who is now a member of e-NABLE and willing to 'Give a Hand'. 


\section{References}

3D Printing Systems. 2015. Up mini 3D Printer. Available: http://store.3dprintingsystems.co.za/UP-Mini-3D-Printer [2015, 25 July].

3DUniverse. 2015. Cyborg Hand Handware Kit. Available: http://shop3duniverse.com/collections/3d-printable-kits/products/e-nable-handassembly-materials-kit-cyborg-beast-edition [2015, 20 July].

Anonymous. Physiotherapist. (Personal Communication, 27 July 2015).

Colombo G, Filippi S, Rizzi C, Rotini F. 2010. A new design paradigm for the development of custom-fit soft sockets for lower limb prostheses. Computers in Industry. 61(6):513-523. DOI:10.1016/j.compind.2010.03.008.

e-NABLE. Cyborg Beast Instruction Manual. Available: http://www.thingiverse.com/thing:261462 [2015, 21 July].

e-NABLE. Frequently Asked Questions. Available: http://enablingthefuture.org/upperlimb-prosthetics/ [2015, 16 July].

e-NABLE. 2015. RIT Arm Assembly Guide. Available: https://www.youmagine.com/designs/rit-e-nable-arm-v0-43\#information [2015, 21 July].

Francis EH, Tay MA, Manna LX, Liu. 2002. A CASD/CASM method for prosthetic socket fabrication using the FDM technology. Rapid Prototyping Journal. 8(4):258262. DOI:10.1108/13552540210441175.

FrederiksI JP, Visagiell S. 2013. The rehabilitation programme and functional outcomes of persons with lower limb amputations at a primary level rehabilitation centre. South African Journal of Occupational Therapy. 43(3):18-27.

Gretsch KF, Lather HD, Peddada KV, Deeken CR, Wall LB, Goldfarb CA. 2015. Development of novel 3D-printed robotic prosthetic for transradial amputees. Prosthetics and Orthotics International. 1-4. DOI:10.1177/0309364615579317. 
Herbert N, Simpson D, Spence W, Ion W. 2005. A preliminary investigation into the development of 3-D printing of prosthetic sockets. Journal of Rehabilitation Research \& Development [serial on the Internet]. 42(2):141-146.

Protolink3d. Printers for Sale. Available: http://protolink3d.co.za/3d-Printer-Sales/ [2015, 24 July].

Rahmati S, Farahmand F, Abbaszadeh F. 2011. Application of rapid prototyping for development of custom-made orthopedics prostheses: an investigative study. International Journal of Advanced Design and Manufacturing Technology. 3(2):11-16.

Rengier F, Mehndiratta A, von Tengg-Kobligk H, Zechmann CM, Unterhinninghofen R, Kauczor HU, Giesel FL. 2010. 3D printing based on imaging data: review of medical applications. International Journal of Computer Assisted Radiology and Surgery. 5:335-341. DOI 10.1007/s11548-010-0476-x.

Robohand. 2015. Introducing Roboleg. [Blog, 22 May] Available: http://www.robohand.net/2014/05/introducing-roboleg/ [2015, 17 July].

Rogers B, Bosker GW, Crawford RH, Faustini MC, Neptune RR, Walden G, Gitter AJ. 2007. Advanced trans-tibial socket fabrication using selective laser sintering. Prosthetics and Orthotics International. 31(1):88-100. DOI:10.1080/03093640600983923.

Smith DG, Burgess EM. 2001. The use of CAD/CAM technology in prosthetics and orthotics-current clinical models and a view to the future. Journal of Rehabilitation Research \& Development. 38(3):327-334.

Zuniga J, Katsavelis D, Peck J, Stollberg J, Petrykowski M, Carson A, Fernandez C. 2015. Cyborg beast: a low-cost 3d-printed prosthetic hand for children with upper-limb differences. BMC Research Notes. 8(10):1-8. DOI:10.1186/s13104-015-0971-9. 